EESTI NSV TEADUSTE AKADEEMIA TOIMETISED. 28. KOIDE FOOSIKA * MATEMAATIKA. 1979, NR. 4

ИЗВЕСТИЯ АКАДЕМИИ НАУК ЭСТОНСКОИ ССР. ТОМ 28 ФИЗИКА * МАТЕМАТИКА. 1979, № 4

\title{
СВЯЗАННЫЕ ВОЛНЫ ДЕФОРМАЦИИ В НЕОДНОРОДНОЙ СРЕДЕ
}

\author{
(Представлена Н. Алумяэ)
}

Классический лучевой метод [ $\left.{ }^{1}\right]$ описывает коротковолновую асимптотику волнового поля в неоднородной среде по линейной модели. С развитием различных модификаций лучевого метода, а вместе с ними и математического аппарата для решения проблем физики плазмы $\left[{ }^{2}\right]$, радиофизики $\left[{ }^{3}\right]$ и нелинейной акустики $\left[{ }^{4}\right]$ стало возможным исследование и нелинейных задач. Однако как в упомянутых, так и в других работах главное внимание уделяется волнам, генерированным краевым воздействием. Покажем здесь, что развитый для анализа нелинейных волн вариант лучевого метода $[2,3,5]$ позволяет учитывать при определении волнового поля и те волны, которые идут в обратном по сравнению с генерированными волнами направлении.

Пусть одномерный переходный волновой процесс деформации в неоднородной среде описывается матричным уравнением

$$
I \partial \mathbf{U} / \partial t+A \partial \mathbf{U} / \partial X+\sum_{p=2} B_{r s} \partial^{p} \mathbf{U} / \partial X^{r} \partial t^{s}+K \partial \mathbf{F} / \partial X+\mathbf{C}=0 .
$$

Здесь $\mathbf{U}$ - неизвестный вектор полевых величин $u_{i}, i=1,2, \ldots, n$; $I$ - единичная матрица; $\mathbf{F}$ - известный вектор, характеризуюший изменение модулей упругости; $A(X, \mathbf{U}), B_{r s}(X, \mathbf{U}), K(X, \mathbf{U})$ - матрицыкоэффициенты, содержащие переменные скорости волн; $\mathbf{C}(X, \mathbf{U})$ вектор, который в зависимости от уравнения состояния может содержать также интегральные операторы. В общем случае уравнение (1) это уравнение смешанного типа, но его главная часть является гиперболической, поскольку обычно выполняется $B_{r s} \sim O(\varepsilon)$, где $\varepsilon-$ малый параметр. Построим для уравнения (1) асимптотическое решение

$$
\mathbf{U}=\sum_{i=0} \varepsilon^{i} \mathbf{U}_{i}
$$

\section{с граничными условиями}

$$
\left.\mathbf{U}(X, t)\right|_{t=0}=0,\left.\mathbf{U}(X, t)\right|_{X=0}=\vec{\Phi}(t)\left[H(t)-H\left(t-t_{0}\right)\right],
$$

где $H(t)$ - функция Хевисайда, $t_{0}$ - продолжительность импульса. Для решения задачи применим модифицированный вариант лучевого метода $[2,3,5]$, который в нелинейной постановке ведет к построению нелинейных уравнений переноса вдоль определенных из ассоциированного гиперболического уравнения бихарактеристик. Будем считать, что выполняются все требования относительно зависимых переменных и коэффициентов уравнения (1) $\left[{ }^{6}\right]$. Индекс «0» далее обозначает первый 
член разложения, причем $A_{0}=A_{0}(X), B_{0 r s}=$ const, $\mathrm{C}_{0}=0$. Тогда ассоциированное уравнение с учетом предположения $\mathrm{F} \sim O(\varepsilon)$ определяет скорости $\lambda_{i}, i=1,2, \ldots, n$, отдельных простых волн. Выбирая лучевые координаты в виде

$$
\xi_{j}=t-\int_{0}^{X}\left(\lambda_{j}(x)\right)^{-1} d x, \quad \tau=\varepsilon X,
$$

получим из уравнения (1) в первом приближении

$$
\sum_{j=1}^{n}\left(I-\lambda-_{j}^{-1} A_{0}\right) \partial U_{0} / \partial \xi_{j}=0
$$

откуда

$$
\mathbf{U}_{0}=\sum_{i=1}^{n} \alpha_{i}\left(\xi_{i}, \tau\right) \mathbf{r}_{i}
$$

Величины $\mathbf{l}_{i}$ и $\mathbf{r}_{i}$ - левые и правые собственные векторы матрицы $A_{0}$ при $\lambda=\lambda_{i}$, а $\alpha_{i}-$ амплитудный фактор. Подчеркнем, что если неоднородность проявляется слабо, тогда $\lambda_{j}=\lambda_{0 j}+O(\varepsilon), \lambda_{0 j}=$ const и взамен (2) можно использовать преобразование

$$
\xi_{j}=\lambda_{0 j} t-X, \quad \tau=\varepsilon X,
$$

которое тоже приводит к соотношению (3). Этот результат свидетельствует о том, что в основу анализа взято разложение по простым волнам. Чтобы облегчить выкладки, построим уравнения переноса для случая слабой неоднородности (4), принимая $\tau_{n}=\varepsilon X_{n} ; X_{n}=X$, если $n=1,3, \ldots$, и $X_{n}=-\left(X-X_{0}\right)$, если $n=2,4, \ldots$. Здесь нечетные $n$ обозначают волны, идущие в сторону нарастания координаты $X$ (направо), а четные $n$ - волны, идущие в сторону убывания координаты $X$ (налево). Уравнение переноса первого порядка в этом случае для каждой $i$-й волны принимает вид

$$
\begin{gathered}
a_{i 0} \partial \alpha_{i} / \partial \tau_{n}+\sum_{j}^{n} a_{j 1} \partial \alpha_{j} / \partial \xi_{j}+\sum_{p=2} a_{p 2} \partial^{p} \alpha_{i} / \partial \xi_{i}^{p}+\sum_{j}^{n} f\left(\alpha_{j}, \tau_{n}\right)=0, \\
a_{i 0}=(-1)^{n+1} \mathbf{l}_{i} A_{0} \mathbf{r}_{i}=(-1)^{n+1} \lambda_{i}, a_{j 1}=-\mathbf{l}_{i} A_{1} \mathbf{r}_{j}, \\
a_{p 2}=-\lambda_{i}^{-1} \mathbf{l}_{i} B_{0 r s} \mathbf{r}_{i}, \quad f\left(\alpha_{j}, \tau_{n}\right)=\mathbf{l}_{i} \mathbf{C}_{1}\left(\mathbf{r}_{j}\right) \alpha_{j}-\lambda_{i}^{-1} \mathbf{l}_{i} K_{0} \partial \mathbf{F}_{0} / \partial \xi_{j} .
\end{gathered}
$$

Структура уравнения (5) показывает, что $i$-я волна зависит и от тех $j$-х волн $(j \in n)$, для которых выполняется $\mathrm{a}_{j 1} \neq 0, f\left(\alpha_{j}, \tau_{n}\right) \neq 0$. Уравнение (5) решается при начальном условии

$$
\left.\alpha_{i}\left(\xi_{i}, \tau_{n}\right)\right|_{\tau_{n}=0}=1_{i} \vec{\Phi}\left(\xi_{i}\right)\left[H(\xi)-H\left(\xi-\xi_{0}\right)\right] .
$$

Рассмотрим теперь конкретную задачу - одномерную задачу полупространства, в котором параметры Лямэ и плотность являются функциями от пространственной координаты. Допустим, что справедливо

$$
\lambda+2 \mu=\left(\lambda_{0}+2 \mu_{0}\right)\left(1+\varepsilon f_{1}(X)\right), \varrho=\varrho_{0}\left(1+\varepsilon h_{1}(X)\right),
$$

где индекс «0» обозначает значение указанной величины в определенной точке (напр., при $X=0$ ) и $\varepsilon-$ малый параметр. Из этих соотношений вытекает также

$$
c^{2}=c_{0}^{2}\left(1+\varepsilon g_{1}(X)\right), \quad g_{1}(X)=f_{1}(X)-h_{1}(X) .
$$


Предположим, что $f_{1}(X)$ и $h_{1}(X)$ являются гладкими функциями, они удовлетворяют условиям $f_{1}(0)=h_{1}(0)=0$ и обладают производными требуемого порядка. Окончательная система уравнений, описывающая распространение продольных волн в полупространстве, получит форму (1), в которой

$$
\begin{gathered}
A=\left|\begin{array}{cc}
0 & c_{0}^{2}\left[1+3\left(1+m_{0}\right) u_{2}+\varepsilon g_{1}(X)\right. \\
-1 & 0
\end{array}\right|, \quad B_{r s}=0, \\
K=\left|\begin{array}{cc}
-c_{0}^{2} u_{2} & 0 \\
0 & 0
\end{array}\right|, \quad \mathbf{U}=\left|\begin{array}{l}
\partial U_{1} / \partial t \\
\partial U_{1} / \partial X
\end{array}\right|, \quad \mathbf{F}=\left|\begin{array}{c}
1+\varepsilon f_{1}(X) \\
0
\end{array}\right|, \\
m_{0}=2\left(v_{1}+v_{2}+v_{3}\right)\left(\lambda_{0}+2 \mu_{0}\right)^{-1} .
\end{gathered}
$$

Здесь $v_{i}, i=1,2,3$ - модули упругости третьего порядка и диссипация для простоты не учитывается. Система уравнений первого порядка (5) принимает теперь вид

$$
\begin{gathered}
\partial \beta_{1} / \partial \sigma_{1}+\operatorname{sign}\left(1+m_{0}\right) \beta_{1} \partial \beta_{1} / \partial \zeta_{1}-\theta(X)\left(\partial \beta_{1} / \partial \zeta_{1}-\partial \beta_{2} / \partial \zeta_{2}\right)+ \\
+\Lambda\left(\beta_{1}-\beta_{2}\right)=0 \\
\partial \beta_{2} / \partial \sigma_{2}-\operatorname{sign}\left(1+m_{0}\right) \beta_{2} \partial \beta_{2} / \partial \zeta_{2}+\theta(X)\left(\partial \beta_{2} / \partial \zeta_{2}-\partial \beta_{1} / \partial \zeta_{1}\right)+ \\
+\Lambda\left(\beta_{1}-\beta_{2}\right)=0 \\
\beta_{i}=\alpha_{i} \alpha_{a}^{-1}, \quad \mathbf{U}_{0}=\sum_{i} \alpha_{i} \mathbf{r}_{i}, \quad \zeta_{1}=\left(c_{0} t-X\right) \tau_{c}^{-1}, \\
\zeta_{2}=\left(c_{0} t+X\right) \tau_{c}^{-1}, \quad \sigma_{1}=\varepsilon X \tau_{c}^{-1}, \quad \sigma_{2}=-\varepsilon\left(X-X_{0}\right) \tau_{c}^{-1}, \\
\theta(X)=\varepsilon c_{0}\left(3\left|1+m_{0}\right| \alpha_{0}\right)^{-1} g_{1}(X), \Lambda=\varepsilon \tau_{c} c_{0}\left(3\left|1+m_{0}\right| \alpha_{0}\right)^{-1} \partial f_{1} / \partial X \\
\mathbf{r}_{1}=\left|\begin{array}{c}
1 \\
-c_{0}^{-1}
\end{array}\right|, \quad \mathbf{r}_{2}=\left|\begin{array}{c}
1 \\
c_{0}^{-1}
\end{array}\right| .
\end{gathered}
$$

Здесь $\tau_{c}-$ длина волны, $\alpha_{0}-$ максимальная амплитуда. Связанность системы (6), (7) является линейной и обусловливается неоднородностью среды. Пусть имеем

$$
\left.\beta_{1}\right|_{\sigma_{t}=0}=\varphi\left(\zeta_{i}\right),\left.\beta_{2}\right|_{\sigma_{t}=0}=0 .
$$

Тогда в силу связанности уравнений (6), (7) по мере роста $\sigma_{1}$ генерируется и волна $\beta_{2}$.

Для решения задачи в явном виде введем в рассмотрение предпосылки, что $\max \beta_{1} \gg \max \beta_{2}, \max \partial \beta_{1} / \partial \zeta_{1} \gg \max \partial \beta_{2} / \partial \zeta_{2}$. Это позволит упростить систему (6), (7):

$$
\begin{gathered}
\partial \beta_{1} / \partial \sigma_{1}+\operatorname{sign}\left(1+m_{0}\right) \beta_{1} \partial \beta_{1} / \partial \zeta_{1}-\theta(X) \partial \beta_{1} / \partial \zeta_{1}+\Lambda \beta_{1}=0, \\
\partial \beta_{2} / \partial \sigma_{2}+\theta(X) \partial \beta_{2} / \partial \zeta_{2}-\Lambda \beta_{2}=\theta(X) \partial \beta_{1} / \partial \zeta_{1}-\Lambda \beta_{1} .
\end{gathered}
$$

Пусть параметры Лямэ изменяются по линейному закону. Тогда имеем $\Lambda=$ const. Из уравнения (8) определяется $\beta_{1}$ и при слабых нелинейных эффектах $\beta_{1}=\beta_{1}\left(\zeta_{1}\right)$. Уравнение (9) допускает запись в характеристиках

$$
\begin{gathered}
d \zeta_{2} / d \sigma_{2}=\theta(X), \\
d \beta_{2} / d \sigma_{2}-\Lambda \beta_{2}=\theta(X) \partial \beta_{1} / \partial \zeta_{1}-\Lambda \beta_{1}
\end{gathered}
$$


Целесообразно в данном случае принять $X_{0}=0$. Из (10) получим теперь

$$
\zeta_{2}-\zeta_{0}=\int_{\sigma_{2}}^{0} \theta(X) d \sigma_{2}
$$

а из (11) вытекает

$$
\beta_{2}=\exp \left(-\Lambda \sigma_{2}\right)\left(\beta_{20}+\int_{\sigma_{2}}^{0}\left(\theta(X) \partial \beta_{1} / \partial \zeta_{1}-\Lambda \beta_{1}\right) \exp \left(\Lambda \sigma_{2}\right) d \sigma_{2}\right) .
$$

Так как $\beta_{2}=0$ при $\zeta_{1}<0$, то $\beta_{20}=0$. С другой стороны, $\beta_{1} \neq 0$ в интервале $\left\{\zeta_{1}=0, \zeta_{1}=\zeta_{1 A}\right\}$. Так как при коротких импульсах можно предполагать $\Delta \xi_{1} \ll \sigma_{2}$, то справедливо

$$
\begin{aligned}
& \int_{\sigma_{2}}^{0}\left(\theta(X) \partial \beta_{1} / \partial \zeta_{1}-\Lambda \beta_{1}\right) \exp \left(\Lambda \sigma_{2}\right) d \sigma_{2}= \\
= & \int_{0}^{\zeta_{1}}\left(\theta(X) \partial \beta_{1} / \partial \zeta_{1}-\Lambda \beta_{1}\right) d \zeta_{1} \int_{\sigma_{2}}^{0} \exp \left(\Lambda \sigma_{2}\right) d \sigma_{2} .
\end{aligned}
$$

При симметричном импульсе выполняется

$$
\int_{0}^{\zeta_{11}} \partial \beta_{1} / \partial \zeta_{1}=0
$$

откуда

$$
\int_{0}^{\zeta_{11}} \beta_{1} d \zeta_{1}=\int_{0}^{\zeta_{1}} \beta_{10} d \zeta_{1}=M_{0}\left(\zeta_{1}\right),
$$

где $M_{0}$ - главный момент импульса $\beta_{1}$. С учетом этих упрощений получим наконец

$$
\beta_{2}=M_{0}\left(1-\exp \left(-\Lambda \sigma_{2}\right)\right)=M_{0}\left(1-\exp \left(\Lambda \tau_{\mathrm{c}}^{-1} \varepsilon X\right)\right) .
$$

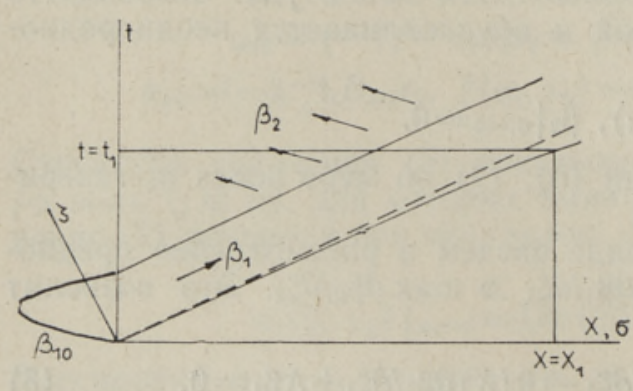

Рис. 1.

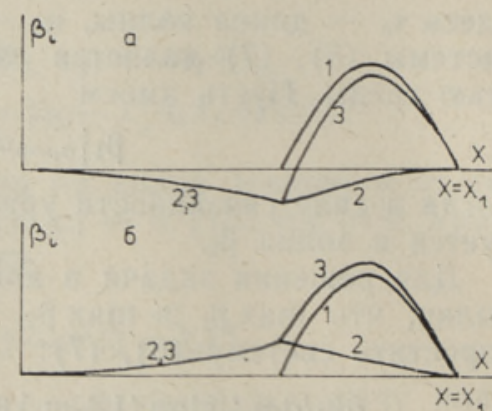

Рис. 2.

На рис. 1 представлена схема процесса на физической плоскости $X, t$, а на рис. 2 показаны профили волн $\beta_{11}$ (кривая 1 ), $\beta_{2}$ (кривая 2) и $\beta_{1}+\beta_{2}$ (кривая 3 ) в момент $t=t_{1}$ согласно решению уравнений (8) и (9). Схема на рис. $2, a$ соответствует случаю $\Lambda>0$, схема на рис. $2, \sigma-$ случаю $\Lambda<0$. Подобная линейная задача, описываемая полной системой (1), была решена в $\left[{ }^{6}\right]$ с помощью преобразования Лапласа. Ре- 
зультаты, полученные здесь лучевым методом, полностью совпадают с результатами точного интегрирования [ $\left.{ }^{6}\right]$. Кроме того, данный подход позволяет учитывать и нелинейность процесса. Как следует из структуры решения $(12), \beta_{2}$ тем больше, чем больше амплитуда волны $\beta_{1}$ (ее главный момент $M_{0}$ ) и чем больше влияние неоднородности (параметр $\Lambda$ ). Необходимо подчеркнуть, что параметр $\Lambda$ зависит от изменения модуля упругости (параметров Лямэ) и приводит к изменению амплитуды. С другой стороны, изменение скорости учитывается в решении $\beta_{i}$ через параметр $\theta$, который обусловливает искривление характеристик на физической плоскости $X, t$ (рис. 1 ).

\section{Л И ТЕРА Т Р А}

1. Ба бич В. М., Булдыре в В. С., Асимптотические методы в задачах дифракции коротких волн. Метод эталонных задач, М., «Наука», 1972.

2. T a ni uti, T., Suppl. Progr. Theor. Phys., № 55, 1-35 (1974).

3. Ост ров ский Л. А., Пелинов ский Е. Н., Прикл. мат. и мех , 38, № 1, 121124. (1974).

4. Руденко О. В., Солуян С. И., Теоретические основы нелинейной акустики, М., «Наука», 1975.

5. Энгельбрехт Ю. К., Изв. АН СССР, Мех. тверд. тела, № 2, 143-148 (1977).

6. Longcope, D. B., Steele, C. R., J. Appl. Mech., E41, № 4, 1057-1062 (1974).

Институт кибернетики

Академии наук Эстонской ССР

Поступила в редакцию

W/VI 1979

\section{J. ENGELBRECHT}

\section{MITTEHOMOGEENSES KESKKONNAS LEVIVAD SEOSTATUD DEFORMATSIOONILAINED}

Modifitseeritud kiirte meetodil on tuletatud seostatud transpordivõrrandite süsteem deformeeruvas keskkonnas levivate ühemōōtmeliste pikilainete kirjeldamiseks. Näitena on vaadeldud juhtu, kus Lamé parameetrite muutus on esitatud lineaarse funktsiooni abil. Sellise juhu tarvis on artiklis toodud lihtne valem, mis kirjeldab vasakule levivat sekundaarset deformatsioonilainet, arvestades ääretingimuse genereeritud paremale levivat primaarset deformatsioonilainet.

\section{J. ENGELBRECHT}

\section{COUPLED DEFORMATION WAVES IN NON-HOMOGENEOUS MEDIUM}

The transient deformation waves in non-homogeneous medium are described using the modified ray method. A system of transport equations is obtained for onedimensional coupled longitudinal waves. An example of deformation wave propagation in solid medium is considered. The longitudinal wave $\beta_{1}$ (propagating to the right) is generated by a bounded boundary iศput. The case when the Lamé parameters change linearly in the space leads to a simple analytic expression (12) for the secondary wave $\beta_{2}$ (propagating to the left). In the expression (12) the following notations are used: $M_{0}$ is the moment of the wave $\beta_{1}, \lambda$ is the coefficient that takes into account the change of the Lamé parameters, $\tau_{c}$ is the effective wave-length, and $\varepsilon$ is the small parameter. The results of the asymptotic analysis are in good coincidence with the exact solution of the linear problem [ $\left.{ }^{6}\right]$. 